\title{
CRISPR/Cas9-mediated deletion of the Wiskott-Aldrich syndrome locus causes actin cytoskeleton disorganization in murine erythroleukemia cells
}

Vanessa Fernández-Calleja ${ }^{1}$ ， María-José Fernández-Nestosa ${ }^{2}$ ， Pablo Hernández ${ }^{1}$ ， Jorge B Schvartzman ${ }^{1}$, Dora B Krimer ${ }^{\text {Corresp. } 1}$

${ }^{1}$ Department of Cellular and Molecular Biology, Centro de Investigaciones Biológicas, Spanish National Research Council (CSIC), Madrid, Spain

Bioinformatic Laboratory, Polytechnic School, National University of Asuncion, San Lorenzo, Paraguay

Corresponding Author: Dora B Krimer

Email address: dbkrimer@cib.csic.es

Wiskott-Aldrich syndrome (WAS) is a recessive X-linked inmmunodeficiency caused by loss-of-function mutations in the gene encoding the WAS protein (WASp). WASp plays an important role in the polymerization of the actin cytoskeleton in hematopoietic cells through activation of the Arp2/3 complex. In a previous study, we found that actin cytoskeleton proteins, including WASp, were silenced in murine erythroleukemia cells defective in differentiation. Here, we designed a CRISPR/Cas9 strategy to delete a 9.5-kb genomic region encompassing the Was gene in the $X$ chromosome of murine erythroleukemia (MEL) cells. We show that Was-deficient MEL cells have a poor organization of the actin cytoskeleton that can be recovered by restoring Was expression. We found that whereas the total amount of actin protein was similar between wild-type and Was knockout MEL cells, the latter exhibited an altered ratio of monomeric G-actin to polymeric F-actin. We also demonstrate that Was overexpression can mediate the activation of Bruton's tyrosine kinase. Overall, these findings support the role of WASp as a key regulator of F-actin in erythroid cells. 
1 Title: "CRISPR/Cas9-mediated deletion of the Wiskott-Aldrich syndrome locus causes actin

2 cytoskeleton disorganization in murine erythroleukemia cells"

3

4 Authors: Vanessa Fernández-Calleja ${ }^{1 \#}$, María-José Fernández-Nestosa ${ }^{2}$, Pablo Hernández $^{1}$, Jorge B. Schvartzman ${ }^{1}$, and Dora B. Krimer ${ }^{1 *}$

6

7 Address: ${ }^{1}$ Department of Cellular and Molecular Biology, Centro de Investigaciones Biológicas,

8 Spanish National Research Council (CSIC), Madrid, Spain, ${ }^{2}$ Bioinformatic Laboratory,

9 Polytechnic School, National University of Asuncion, San Lorenzo, Paraguay

10

11 Running Title: Wiskott-Aldrich syndrome locus knockout in erythroleukemia cells

12

${ }^{*}$ Corresponding author: Dora B. Krimer

14

Address: CIB, CSIC, Ramiro de Maeztu 9, 28040-Madrid, Spain, Phone: (+34) 918373112 ext.

15

4238, Fax: (+34) 91536 0432, E-mail: dbkrimer@cib.csic.es

16

17 \#Present address: Centro de Biotecnología y Genómica de Plantas (UPM-INIA), Campus

18 Montegancedo, Madrid, Spain

19

20

21

22 
24 ABSTRACT

25

26 Wiskott-Aldrich syndrome (WAS) is a recessive X-linked immunodeficiency caused by loss-of-

27 function mutations in the gene encoding the WAS protein (WASp). WASp plays an important

28 role in the polymerization of the actin cytoskeleton in hematopoietic cells through activation of

29 the Arp2/3 complex. In a previous study, we found that actin cytoskeleton proteins, including

30 WASp, were silenced in murine erythroleukemia cells defective in differentiation. Here, we

31 designed a CRISPR/Cas9 strategy to delete a 9.5-kb genomic region encompassing the Was gene

32 in the X chromosome of murine erythroleukemia (MEL) cells. We show that Was-deficient MEL

33 cells have a poor organization of the actin cytoskeleton that can be recovered by restoring Was

34 expression. We found that whereas the total amount of actin protein was similar between wild-

35 type and Was knockout MEL cells, the latter exhibited an altered ratio of monomeric G-actin to 36 polymeric F-actin. We also demonstrate that Was overexpression can mediate the activation of

37 Bruton's tyrosine kinase. Overall, these findings support the role of WASp as a key regulator of 38 F-actin in erythroid cells.

39

40

41

42

43

44 45 
46 Wiskott-Aldrich syndrome (WAS) is an X-linked hematological disorder clinically characterized

47 by microthrombocytopenia, eczema, recurrent infections and predisposition to develop

48 lymphomas and autoimmunity diseases (Massaad et al. 2013; Matalon et al. 2013; Thrasher \&

49 Burns 2010). The disease arises from mutations in the gene encoding the WAS protein (WASp),

50 resulting in cytoskeletal abnormalities that are responsible for the wide spectrum of clinical

51 phenotypes. The levels of WASp expression correlate negatively with the severity of the disease;

52 accordingly, low levels produce milder forms such as X-linked thrombocytopenia, whereas the

53 absence of the protein results in the most serious manifestations of Wiskott-Aldrich syndrome

54 (Albert et al. 2010; Jin et al. 2004; Zhu et al. 1997).

55

56 WASp is the founder member of a family of actin nucleation-promoting factors that includes at

57 least 5 subfamilies, WASP (WASP and neuronal N-WASP), SCAR/WAVE (verprolin homolog

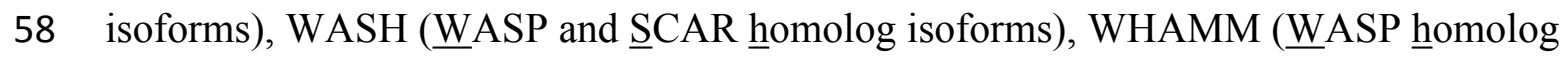

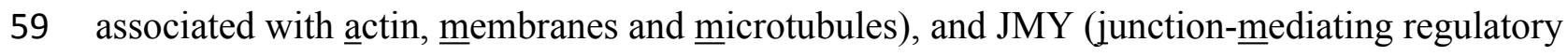

60 protein) (Alekhina et al. 2017), and all are important regulators of actin cytoskeletal dynamics.

61 WASp family proteins function as multidomain proteins that adopt a closed autoinhibited

62 conformation, where the carboxy-terminal verprolin-cofilin-acidic domain interacts with the

63 GTPase-binding domain. Binding of the Rho GTPase Cdc42 protein unlocks the verprolin-

64 cofilin-acidic region, allowing binding and activation of the Arp2/3 (actin-related protein)

65 complex, which subsequently stimulates actin polymerization (Kim et al. 2000). As opposed to

66 the ubiquitous expression of other WASp family members, WASp is exclusively expressed in the

67 hematopoietic lineage (Derry et al. 1994; Parolini et al. 1997), including erythroid cells

68 (Fernandez-Calleja et al. 2017; Parolini et al. 1997), 
69

70 The eukaryotic actin cytoskeleton is a key element in various cellular processes and is classically

71 associated with cell migration, adhesion, endo/exocytosis, and cytokinesis. Actin microfilaments

72 are composed of monomeric globular (G-actin) and polymeric, filamentous actin (F-actin), and

73 the dynamic transition between the two forms are dependent on the cellular requirements (Rotty

$74 \&$ Bear 2014). Increasing data show that actin dynamics is also involved in signaling regulation

75 and plays important roles in cell differentiation (Misu et al. 2017) and tumor progression (Ebata

76 et al. 2016; Nurnberg et al. 2011). We recently demonstrated that the actin cytoskeleton is poorly

77 organized in a murine erythroleukemia (MEL) cell line resistant to cell differentiation

78 (Fernandez-Calleja et al. 2017). Unlike the progenitor MEL-DS19 cell line, resistant cells (MEL-

79 R) are refractive to most inducers capable of triggering cell differentiation (Fernandez-Nestosa et

80 al. 2008; Fernandez-Nestosa et al. 2013). Transcriptome profiling by next-generation sequencing

81 of MEL-DS19 and MEL-R cell lines revealed that several genes involved in actin polymerization

82 were poorly expressed in MEL-R cells. WASp emerged as one of the actin-related proteins

83 whose expression was blunted in resistant cells (Fernandez-Calleja et al. 2017). Accordingly,

84 MEL-R cells showed a marked decrease in actin content as measured by immunocytochemistry

85 and confocal microscopy, even though the total amount of actin protein remained unchanged.

86 Based on these observations, we suggested that actin-related proteins might shape the

87 cytoskeleton organization. Furthermore, we hypothesized that the loss of any of the actin-

88 network components may interfere with the dynamic assembly that takes place during actin

89 polymerization. In the present study, we used CRISPR/Cas9 to delete the Was locus in MEL

90 DS19 cells. We found that loss of WASp altered the dynamics of filamentous actin (F-actin) and

91 free globular actin (G-actin) turnover, which led to an aberrant actin cytoskeleton organization. 
92 The phenotype displayed by the CRISPR/Cas9-edited Was transfectants resembled that of MEL-

93 R cells, and could be recovered by WASp overexpression. We also show that ectopic expression

94 of WASp enhances the expression of Bruton's tyrosine kinase, an important component of the

95 actin cytoskeleton network.

96

97

98

99

100

101

102

103

104

105

106

107

108

109

110

111

112 counting samples of the cultures with a Neubauer hemocytometer chamber.

\section{MATERIALS AND METHODS}

\section{Cell cultures} from MEL cells, were previously established in our laboratory by growing MEL cells continuously in the presence of $5 \mathrm{mM}$ hexamethylene bisacetamide (HMBA) (FernandezMedium containing 10\% fetal bovine serum (BSA), 100 units $/ \mathrm{ml}$ penicillin and $100 \mu \mathrm{g} / \mathrm{ml}$ streptomycin (all from Gibco). MEL-R cells were routinely cultured in the presence of $5 \mathrm{mM}$ of benzidine-staining positive cells $(\mathrm{B}+)$ in cell cultures. Cell growth was assessed daily by

The murine erythroleukemia cell line MEL-DS19 (hereafter called MEL) was obtained from Dr Arthur Skoultchi (Albert Einstein College of Medicine, New York, USA). MEL-R cells, derived Nestosa et al. 2008; Fernandez-Nestosa et al. 2013). Murine 3T3-Swiss albino fibroblasts (CCL92) were obtained from the ATTC. Cell lines were propagated in Dulbecco's Modified Eagle's HMBA (Sigma). MEL DS19 cell differentiation was induced by exposing exponentially growing cultures to $5 \mathrm{mM}$ HMBA. Hemoglobinized cells were quantified by determining the proportion

114 Generation of MEL/Was ${ }^{-/-}$cells by CRISPR/Cas9 technology 
115 Genomic deletion of Was in MEL cells was performed by CRISPR/Cas9 technology as described

116 (Bauer et al. 2015). Two single guide RNAs (sgRNA1 and sgRNA2) were designed to separately

117 target the entire gene (mouse X:7658591-7667617) using the online-tool http://crispr.mit.edu/.

118 Coupled complementary oligonucleotides (CACC was added to the 5' end of the sense strand

119 and AAAC was added to the 5' end of the antisense strand) were annealed and inserted into

120 the BbsI sites of linearized pX330 vector (Addgene plasmid ID 42230). The sequences of the

121 sgRNA oligonucleotides are listed in Table S1. MEL cells were co-transfected with the two

122 sgRNAs vectors and a third vector, pEFBOS-GFP, encoding green fluorescent protein (GFP)

123 (Fig. S1), by cationic liposome-based transfection with Lipofectamine 2000 (Life Technologies).

124 After 72 hours, the top $\sim 3 \%$ of GFP-positive cells were individually sorted into 96-well plates.

125 Genomic DNA was isolated from all clones and screened for biallelic deletion via PCR using

126 non-deletion (ND) primers, whose assembly takes place internal to the sequence to be deleted,

127 and deletion (D) primers, whose assembly is upstream and downstream of the sgRNA cleavage

128 sites. The primers used for identifying biallelic deletion clones are listed in Table S2 and were

129 designed with Primer3 software (http://bioinfo.ut.ee/primer3-0.4.0/) (Untergasser et al. 2012).

130 The conditions for the PCR were as follows: pre-denaturing step of $94^{\circ} \mathrm{C}$ for $7 \mathrm{~min}$, followed by

13135 cycles of $94^{\circ} \mathrm{C}$ for $40 \mathrm{sec}, 60^{\circ} \mathrm{C}$ for $1 \mathrm{~min}$ and $72^{\circ} \mathrm{C}$ for $1 \mathrm{~min}$, with a final extension at $72^{\circ} \mathrm{C}$

132 for 7 min. PCR products were resolved on 1\% agarose gels and visualized by ethidium bromide 133 staining.

\section{MEL-R DNA transfection}

136 Exponentially growing MEL-R cells were transfected as described above with the

137 pcDNA3.1 \pm DYK Was expression vector (GeneScript) containing the coding region of Was 
138 (hereafter called pcDNA3.1-Was) (Fig. S1). After 6 hours, cells were distributed into 96-well

139 plates. The transfectants were selected by limited dilution and maintained in growth medium

140 containing $1 \mu \mathrm{g} / \mathrm{ml} \mathrm{G} 418$ (Sigma).

141

\section{Antibodies and immunoblotting}

143 Control 3T3 fibroblast cells, MEL, MEL-R and transfected cells $\left(2.5 \times 10^{6}\right)$ were harvested, 144 washed with phosphate buffered saline (PBS) and lysed with NP-40 buffer (20 mM Tris-HCl pH $1457.5,10 \%$ glycerol, $137 \mathrm{mM} \mathrm{NaCl}, 1 \% \mathrm{NP}-40,1 \mathrm{mM}$ sodium orthovanadate, $10 \mathrm{mM}$ sodium 146 fluoride, and 2 mM EDTA) containing protease inhibitors (all from Sigma). Protein lysates (10$14730 \mu \mathrm{g}$ ) were separated by $12 \%$ SDS-polyacrylamide gel electrophoresis and transferred to PVDF 148 membranes (Bio-Rad). The membranes were incubated with a mouse monoclonal anti- $\beta$-actin 149 (1:10000, Sigma), mouse monoclonal anti-WASp (1:500, Santa Cruz), mouse monoclonal anti150 Btk (1:500, Santa Cruz), and rabbit polyclonal anti- $\alpha$-tubulin (1:1000, ABclonal) antibodies, then 151 washed five times with T-TBS (20 mM Tris-HCl, $150 \mathrm{mM} \mathrm{NaCl}, 0.1 \%$ Tween 20). Primary 152 antibodies were detected with horseradish peroxidase-conjugated anti-mouse (1:3000, Santa 153 Cruz) or anti-rabbit IgG (1:1000, DAKO) antibodies, followed by five cycles of T-TBS washes. 154 The analysis of filamentous (F-actin) and globular (G-actin) actin content was performed from $15510^{7}$ cells. Samples were harvested, washed in PBS and lysed in a lysis buffer (50 mM PIPES pH 156 6.9 (Sigma), $5 \mathrm{mM} \mathrm{MgCl} 2,5 \mathrm{mM}$ EGTA (Sigma), $5 \%$ glycerol (Roche), $0.1 \% \beta-$

157 mercaptoethanol (Merck), $1 \mathrm{mM}$ PMSF (Roche), $10 \mathrm{mM}$ benzamidine (Sigma) and $1 \mathrm{mM}$ ATP

158 (Roche)). Protein supernatants and pellets were collected after ultracentrifugation (100,000 g, $1 \mathrm{~h}$ 159 at $37^{\circ} \mathrm{C}$ ) and analyzed by immunoblotting as described above. 


\section{Immunofluorescence staining and confocal microscopy}

162 MEL, MEL-R and transfected cells were plated on poly-L-lysine-coated slides and incubated at

$16337^{\circ} \mathrm{C}$ for $30 \mathrm{~min}$. Cells were fixed with $4 \%$ paraformaldehyde for $30 \mathrm{~min}$, permeabilized with

$1640.1 \%$ Triton-X 100 in PBS for 30 min and blocked with 1\% BSA in PBS/0.1\% Triton-X 100 for

$1651 \mathrm{~h}$, all at room temperature $\left(\mathrm{RT}, \sim 22^{\circ} \mathrm{C}\right)$. Cells were stained with anti- $\beta$-actin $(1: 3000$, Sigma $)$ or

166 anti-Btk (1:200, Santa Cruz) antibodies for $1 \mathrm{~h}$ at RT followed by washing twice with PBS.

167 Primary antibodies were detected with an Alexa Fluor 568 secondary antibody (Molecular

168 Probes) and $1 \mu \mathrm{g} / \mathrm{ml}$ DAPI (4,6-diamidino-2-phenylindole, Sigma) was added to stain nuclei, for 169 1h at RT, followed by two washes with PBS. Finally, cells were mounted on cover slips with

170 Prolong Diamond Antifade Mountant reagent (Invitrogen). Fluorescence images were acquired

171 on a Leica TCS SP2 confocal microscope using a 100× objective with zoom.

172

173 Statistical analysis

174 Data are presented as means \pm standard deviation of the densitometric analysis. Differences were

175 tested by the Student t-test. The values $\mathrm{P}<0.05$ were considered statistically significant.

176 Statistical analysis of western blot data and immunofluorescence images are presented in Fig.S2

177 and Fig.S3, respectively.

178

179

180

RESULTS

181

182 Effects of ectopic WASp expression on the actin cytoskeleton in MEL-R cells 
184 It is generally recognized that WASp plays an important role in the maintenance of cytoskeletal 185 organization in hematopoietic cells (Alekhina et al. 2017). In our previous study, we found that 186 Was is expressed in erythroleukemia MEL cells, whereas it was barely detectable in MEL-R cell 187 lines (Fernandez-Calleja et al. 2017). To further evaluate the status of Was at the protein level, 188 we compared proteins extracts from MEL cells, both undifferentiated and differentiated in the 189 presence of the inducer-mediated differentiation HMBA, with those from a representative MEL$190 \mathrm{R}$ resistant line. We also included the $3 \mathrm{~T} 3$ fibroblast cell line as a negative control as WASp is 191 not expressed in non-hematopoietic lineages. Immunoblotting showed robust WASp expression 192 in MEL cells, which decreased during cell differentiation (Fig. 1A). By contrast, the levels of 193 WASp in MEL-R cells were much lower than in undifferentiated MEL cells and were 194 comparable with that observed in 3T3 fibroblasts. Because our recent study (Fernandez-Calleja 195 et al. 2017) suggested that low levels of several actin-cytoskeletal proteins, including WASp, 196 result in anomalous cytoskeleton organization, we sought to determine whether forced 197 expression of WASp might reverse this effect. To do this, we established MEL-R cell lines 198 constitutively expressing WASp and screened for clones with robust WASp expression, which 199 identified clones 9, 10 and 11 for further analysis (Fig. 1B).

200

201 Confocal immunofluorescence analysis of MEL cells stained with an anti-actin antibody 202 revealed a clear rim of actin fluorescence surrounding the nuclear periphery (Fig. 2, column 1). 203 A similar pattern was observed in the WASp-overexpressing MEL-R clones 9, 10 and 11 (Fig. 2, 204 columns 2-4), whereas no detectable signal was evident in non-Wasp-expressing MEL-R cells 205 (Fig. 2, column 5). These results confirm that WASp expression has a positive impact on the 206 organization of the actin cytoskeleton in erythroleukemia cells. 
208 We next used immunoblotting to evaluate total actin protein levels in MEL-R and WASp209 overexpressing MEL-R transfectants, finding no differences between the two (Fig. 3A). Given

210 this result, we next asked whether the changes observed by confocal microscopy might be due to 211 an altered ratio of monomeric G-actin and polymeric F-actin. To address this, we used high-

212 speed centrifugation to separate G- and F-actin pools from cell lysates, followed by

213 immunoblotting with an anti- $\beta$ actin antibody. Results showed that the F-actin pool in WASp-

214 overexpressing MEL-R clones 9, 10 and 11 was considerably greater than in MEL-R cells, 215 whereas the free G-actin pool remained low (Fig. 3B). By contrast, the G- and F-actin pools in 216 control MEL-R cells were similar. These results demonstrate that the proportion of F-actin 217 increases after WASp overexpression and suggest that it might contribute to nuclear actin 218 polymerization.

\section{CRISPR/Cas9-mediated Was deletion in MEL cells}

221

222 Proteins associated with the actin polymerization machinery, such as WASp, are silenced or 223 minimally expressed in MEL-R cells (Fernandez-Calleja et al. 2017). We have shown that 224 ectopic expression of WASp in MEL-R cells can restore the wild-type phenotype observed for 225 the actin cytoskeleton (Fig. 2). Assuming that WASp is essential for actin polymerization in 226 erythroleukemia cells, we hypothesized that the knockout of the gene would alter actin 227 cytoskeleton organization in MEL cells. Thus, we used the CRISPR/Cas9 gene-editing platform 228 to delete the genomic region encompassing the Was gene in the X chromosome of MEL cells. A 229 schematic representation of the genomic target sites is shown in Fig. 4A. Cells were transfected 
230 with 3 plasmids: pX330Was 1 and pX330Was2, expressing the sgRNAs, and pEFBOS-GFP (Fig.

231 S1). Cells were then double selected for GFP-positivity and G418 resistance. PCR analysis of

232 bi-allelic GFP-positive cells (Fig. 4B) and immunoblotting (Fig. 4C) confirmed three knockout

233 clones: 1, 4 and 73. Cell clone-1 was used in most of the following experiments.

234

235 We first assessed whether Was knockout (MEL/Was ${ }^{-/}$) cells had any noticeable phenotypic

236 differences compared with wild-type MEL cells. Cell proliferation analysis showed no

237 significant alterations in growth rate between MEL and MEL/Was ${ }^{-/-}$cells (Fig. S4A). Cell

238 differentiation was also evaluated after treatment with $5 \mathrm{mM}$ HMBA, a potent inducer of cell

239 differentiation in erythroleukemia cells (Fig. S4B). Results showed no changes in the percentage

240 of cell differentiation between the two cultures, indicating that CRISPR/Cas9-mediated deletion

241 had no deleterious effects on cell transfectants.

242

243 We then questioned whether MEL/Was ${ }^{-/-}$cells presented a defect in the actin cytoskeleton

244 organization. By confocal analysis, loss of $\mathrm{Was}$ function in MEL/Was ${ }^{-/-}$cells led to a

245 considerably reduced actin fluorescence signal, which was similar in intensity to that observed in

246 MEL-R cells (Fig. 5 compare columns 2 and 4). Since the ectopic overexpression of Was in

247 MEL-R cells could rescue the wild-type phenotype (Fig. 2), we performed a similar analysis in

$248 \mathrm{MEL} / \mathrm{Was}^{-/-}$cells. We transiently transfected the pcDNA3.1-Was vector into MEL/Was ${ }^{-/-}$cells

249 and collected cells $48 \mathrm{~h}$ later for immunofluorescence analysis. As expected, a rim of actin

250 staining was clearly visible around the nuclei of MEL/Was $\mathrm{W}^{-/-}$cells overexpressing $\mathrm{Was}$, and was

251 comparable with that observed in MEL cells (Fig. 5, compare columns 3 and 1). Overall, the 
252 knockout and rescue experiments confirm the important effect of WASp for the actin

253 cytoskeleton organization in erythroleukemia cells.

254

255 To examine whether changes in actin expression or an altered ratio of monomeric G-actin and

256 polymeric F-actin was the origin of the actin phenotype in MEL-Was ${ }^{-/-}$cells, we first measured

257 the expression of actin in MEL-Was ${ }^{-/-}$cells by immunoblotting We found that the total amount of

258 actin was similar to that in MEL and MEL-R cell lines (Fig. 6A). We then used

259 ultracentrifugation to fractionate monomeric G-actin to the supernatant and polymeric F-actin to

260 the pellet from MEL-Was ${ }^{-/}$whole extracts and immunoblotted these fractions against actin.

261 Results showed that the balance between G- and F-actin shifted towards G-actin, with markedly

262 less F-actin detected in the pellet of the Was mutant (Fig. 6B).

263

264 Ectopic expression of WASp promotes Btk activation in MEL-R and MEL/Was ${ }^{-/-}$cells

265 There is increasing evidence that actin is also present in the cell nucleus, which is referred to as

266 "nuclear actin", where it participates in transcriptional activation and chromatin remodeling (for

267 a recent review see (Misu et al. 2017)). Sadhukan and co-workers previously demonstrated a

268 nuclear role for WASp in transcriptional activation of several master genes during Th1 cell

269 differentiation, a role that is independent of actin polymerization (Sadhukhan et al. 2014). In a

270 different study, WASp was reported to be present both in the cytoplasm and the nucleus, and

271 regulated gene transcription in K562 myeloid cells (Looi et al. 2014). Because WASp can

272 physically associate with Bruton's tyrosine kinase (Btk) (Baba et al. 1999; Sakuma et al. 2012),

273 and since Btk was included among the silenced actin-associated proteins in MEL-R cells

274 (Fernandez-Calleja et al. 2017), we finally asked whether the ectopic expression of Was affects 
275 Btk expression. Figure 7 shows the results of immunoblotting of whole-cell lysates from the

276 stable WASp-overexpressing MEL-R transfectants 9, 10 and 11 against an anti-Btk antibody. We

277 observed that Btk was expressed at higher levels in all three transfectants than in MEL-R cells,

278 although it was more evident in clones 10 and 11 . We also analyzed MEL-Was ${ }^{-/-}$cells (clones 1

279 and 73), before and after enforced expression of WASp. In all cases, Btk was expressed at a level

280 similar to that of the MEL progenitor line irrespective of whether Wasp was overexpressed or

281 not (Fig. 7). These results reveal that Was overexpression can mediate Btk activation in MEL-R

282 cells, suggesting that Btk is downstream of Was in the signaling cascade. By contrast, an

283 alternative signaling pathway might be operative in MEL cells with targeted deletion of Was.

284 Indeed, Btk expression can be modulated by different upstream activators, such as PU.1 (Christie

285 et al. 2015; Himmelmann et al. 1996). We confirmed these results by confocal microscopy,

286 observing a marked increase in the levels of Btk antibody staining in all three MEL-R clones

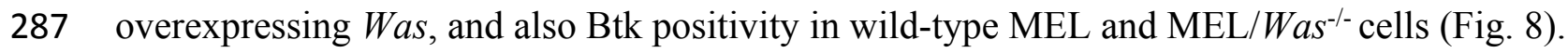

288

289

290 DISCUSSION

291

292 Our previous work aimed to profile differentially-expressed genes between progenitor MEL cells 293 and the derived cell line MEL-R, with induced resistance to differentiation (Fernandez-Calleja et 294 al. 2017). We identified a group of genes comprising three main features: down-regulated in the 295 resistant cell line, preferentially expressed in the hematopoietic lineage, and implicated in the 296 actin cytoskeleton organization. Interestingly, several of these genes, for example, Was, Btk and

297 Rac2, give rise to severe phenotypes when mutated in humans (Ambruso et al. 2000; Bosticardo 
298 et al. 2009; Conley et al. 2009). In the present study, we investigated the influence of Was

299 expression on the organization of the actin cytoskeleton in murine erythroleukemia cells. A

300 previous study by Symons and co-workers using transient transfection of WASp-expressing

301 vectors demonstrated that WASp has a profound effect on actin polymerization in rat kidney

302 epithelial, monkey COS7 kidney and Jurkat T cells (Symons et al. 1996). Furthermore,

303 retrovirus-mediated expression of WASp was shown to reconstitute the actin cytoskeleton in

304 human hematopoietic stem cells and myeloid derivative cells as well as in T and B cells and

305 macrophages (Dewey et al. 2006) and references therein). Our results show that forced

306 expression of WASp in the erythroid lineage MEL-R cell line helped to rebuild actin

307 cytoskeleton organization. At the molecular level, we found that WASp overexpression induced

308 the conversion of G- to F-actin, which resulted in and increased concentration of F-actin. WASp

309 expression is crucial for actin filament nucleation, a task that is carried out through the regulation

310 of the Arp2/3 complex (Machesky \& Insall 1998). A classical view of actin homeostasis involves

311 the ratio of G-/F-actin that is coordinated through signaling cascades (Blanchoin et al. 2014;

312 Burke et al. 2014). The constant competition for a limited pool of actin monomers is tightly

313 controlled by regulatory factors such as profilin-1, which in turn antagonizes WASp (Rotty et al.

314 2015; Suarez et al. 2015). By using CRISPR/Cas9 genome editing in MEL cells, we

315 corroborated that the suppression of WASp has a deleterious effect on the actin cytoskeleton.

316 Furthermore, the G-/F-actin ratio reflects a critical imbalance at the expense of the filamentous

317 component. Taken together, our results suggest that WASp deficiency impedes correct

318 cytoskeleton organization likely by blocking F-actin polymerization. 
320 Phosphorylation of WASp is crucial for multiple cellular responses (Blundell et al. 2009). Btk is

321 a non-receptor kinase that can phosphorylate WASp and relieve the autoinhibitory conformation

322 that ultimately blocks actin polymerization. Btk specifically phosphorylates tyrosine 293 (Y293

323 in mice, Y291 in humans) within the GTPase binding domain, which triggers the destabilization

324 of the autoinhibited conformation and facilitates binding of the Arp2/3 complex (Blundell et al.

325 2009). Sakuma and co-workers demonstrated that the interaction between the WASp N-terminal

326 domain and the SH3 domain of Btk plays important roles in the lipopolysaccharide-TLR4

327 signaling cascade in macrophages (Sakuma et al. 2015). Most of the studies concerning the

328 interaction between WASp and Btk indicate that WASp is located downstream of Btk, as first

329 suggested for B cell development (Baba et al. 1999). Our findings indicate that, directly or

330 indirectly, WASp modulates the expression of Btk. Beyond WASp, other factors can act on Btk,

331 as is the case of the transcription factor PU.1 (Christie et al. 2015; Himmelmann et al. 1996).

332 PU.1 is expressed in MEL cells, whereas it is silenced in MEL-R cells. A possible reason why

333 the expression of Btk was not altered in MEL/Was ${ }^{-/}$cells might be that PU.1 has redundant

334 activity. In MEL-R cells, however, the absence of both WASp and PU.1 would prevent the

335 activation of Btk. Indeed, as we show here, restoration of WASp activates Btk. These seemingly

336 contradictory findings suggest that the interactions governing WASp and Btk, and probably other

337 members of the actin network, are complex.

338

339 Finally, what would be the importance of actin cytoskeleton-related proteins during

340 erythropoiesis? MEL cells, and their MEL-R variants are derived from proerythroblasts infected

341 with the Friend virus complex. When treated with inducers of differentiation, MEL cells can

342 complete the differentiation program and reach the reticulocyte stage. Under specific conditions, 
343 when grown in the presence of a fibronectin matrix, a large proportion of MEL cells will

344 complete enucleation (Patel \& Lodish 1987). MEL-R cells, however, are unable to grow on

345 fibronectin-coated plates and cannot complete enucleation loss (Fernandez-Nestosa 2007).

346 Enucleation is a complex process that mirrors cytokinesis and, accordingly, the actin

347 cytoskeleton and the multiple proteins that make it up have a fundamental role (Konstantinidis et

348 al. 2012). The deregulation of these proteins could negatively influence and prevent enucleation.

349

350

351 CONCLUSIONS

352

353 In the present work, we used CRISPR/Cas9 to delete the Was gene in erythroleukemia cells. We

354 show that Was-deficient cells have a poor organization of the actin cytoskeleton that is

355 accompanied by an imbalance in the ratio of monomeric G- and polymeric F-actin; a defect that

356 is reversed by restoring Was expression. We also demonstrate that Was overexpression mediates

357 the activation of another member of the actin cytoskeleton network, Bruton's tyrosine kinase.

358 Overall, our results support the role of WASp as a key mediator of F-actin regulation and

359 illustrate its importance in the erythroid lineage.

360

361 ACKNOWLEDGEMENTS

362 We thank members of the "Microtubule stabilizing agents" group, J-Fernando Díaz and Daniel

363 Lucena-Agell for their technical advice on ultracentrifugation and G-/F-actin-separation

364 protocols. We acknowledge Mayte Dominguez and Gemma Rodriguez for their help with

365 confocal images analysis and Alicia Bernabé for technical help. 


\section{REFERENCES}

369 Albert MH, Bittner TC, Nonoyama S, Notarangelo LD, Burns S, Imai K, Espanol T, Fasth A, 370 Pellier I, Strauss G, Morio T, Gathmann B, Noordzij JG, Fillat C, Hoenig M, Nathrath M, 371 Meindl A, Pagel P, Wintergerst U, Fischer A, Thrasher AJ, Belohradsky BH, and Ochs HD. 372 2010. X-linked thrombocytopenia (XLT) due to WAS mutations: clinical characteristics, long373 term outcome, and treatment options. Blood 115:3231-3238. 10.1182/blood-2009-09-239087

374 Alekhina O, Burstein E, and Billadeau DD. 2017. Cellular functions of WASP family proteins at 375 a glance. J Cell Sci 130:2235-2241. 10.1242/jcs.199570

376 Ambruso DR, Knall C, Abell AN, Panepinto J, Kurkchubasche A, Thurman G, Gonzalez-Aller

377 C, Hiester A, deBoer M, Harbeck RJ, Oyer R, Johnson GL, and Roos D. 2000. Human 378 neutrophil immunodeficiency syndrome is associated with an inhibitory Rac2 mutation. Proc 379 Natl Acad Sci U S A 97:4654-4659.

380 Baba Y, Nonoyama S, Matsushita M, Yamadori T, Hashimoto S, Imai K, Arai S, Kunikata T, 381 Kurimoto M, Kurosaki T, Ochs HD, Yata J, Kishimoto T, and Tsukada S. 1999. Involvement of 382 wiskott-aldrich syndrome protein in B-cell cytoplasmic tyrosine kinase pathway. Blood 93:20033832012.

384 Bauer DE, Canver MC, and Orkin SH. 2015. Generation of genomic deletions in mammalian cell 385 lines via CRISPR/Cas9. J Vis Exp:e52118. 10.3791/52118

386 Blanchoin L, Boujemaa-Paterski R, Sykes C, and Plastino J. 2014. Actin dynamics, architecture, 387 and mechanics in cell motility. Physiol Rev 94:235-263. 10.1152/physrev.00018.2013 
388 Blundell MP, Bouma G, Metelo J, Worth A, Calle Y, Cowell LA, Westerberg LS, Moulding DA, 389 Mirando S, Kinnon C, Cory GO, Jones GE, Snapper SB, Burns SO, and Thrasher AJ. 2009.

390 Phosphorylation of WASp is a key regulator of activity and stability in vivo. Proc Natl Acad Sci

391 US A 106:15738-15743. 10.1073/pnas.0904346106

392 Bosticardo M, Marangoni F, Aiuti A, Villa A, and Grazia Roncarolo M. 2009. Recent advances 393 in understanding the pathophysiology of Wiskott-Aldrich syndrome. Blood 113:6288-6295.

394 Burke TA, Christensen JR, Barone E, Suarez C, Sirotkin V, and Kovar DR. 2014. Homeostatic 395 actin cytoskeleton networks are regulated by assembly factor competition for monomers. Curr 396 Biol 24:579-585. 10.1016/j.cub.2014.01.072

397 Christie DA, Xu LS, Turkistany SA, Solomon LA, Li SK, Yim E, Welch I, Bell GI, Hess DA, 398 and DeKoter RP. 2015. PU.1 opposes IL-7-dependent proliferation of developing B cells with 399 involvement of the direct target gene bruton tyrosine kinase. J Immunol 194:595-605.

400 Conley ME, Dobbs AK, Farmer DM, Kilic S, Paris K, Grigoriadou S, Coustan-Smith E, Howard 401 V, and Campana D. 2009. Primary B cell immunodeficiencies: comparisons and contrasts. Annu 402 Rev Immunol 27:199-227.

403 Derry JM, Ochs HD, and Francke U. 1994. Isolation of a novel gene mutated in Wiskott-Aldrich 404 syndrome. Cell 79:following 922.

405 Dewey RA, Avedillo Diez I, Ballmaier M, Filipovich A, Greil J, Gungor T, Happel C, Maschan 406 A, Noyan F, Pannicke U, Schwarz K, Snapper S, Welte K, and Klein C. 2006. Retroviral WASP 407 gene transfer into human hematopoietic stem cells reconstitutes the actin cytoskeleton in myeloid 408 progeny cells differentiated in vitro. Exp Hematol 34:1161-1169. 10.1016/j.exphem.2006.04.021 409 Ebata T, Hirata H, and Kawauchi K. 2016. Functions of the Tumor Suppressors p53 and Rb in 410 Actin Cytoskeleton Remodeling. Biomed Res Int 2016:9231057. 10.1155/2016/9231057 
411 Fernandez-Calleja V, Hernandez P, Schvartzman JB, Garcia de Lacoba M, and Krimer DB.

412 2017. Differential gene expression analysis by RNA-seq reveals the importance of actin

413 cytoskeletal proteins in erythroleukemia cells. PeerJ 5:e3432. 10.7717/peerj.3432

414 Fernandez-Nestosa MJ. 2007. Desbloqueo de la diferenciación en células eritroleucémicas

415 resistentes a los agentes inductores PhD. Universidad Complutense de Madrid

416 Fernandez-Nestosa MJ, Hernandez P, Schvartzman JB, and Krimer DB. 2008. PU.1 is

417 dispensable to block erythroid differentiation in Friend erythroleukemia cells. Leuk Res 32:121418130.

419 Fernandez-Nestosa MJ, Monturus E, Sanchez Z, Torres FS, Fernandez AF, Fraga MF,

420 Hernandez P, Schvartzman JB, and Krimer DB. 2013. DNA methylation-mediated silencing of

421 PU.1 in leukemia cells resistant to cell differentiation. Springerplus 2:392.

422 Himmelmann A, Thevenin C, Harrison K, and Kehrl JH. 1996. Analysis of the Bruton's tyrosine 423 kinase gene promoter reveals critical PU.1 and SP1 sites. Blood 87:1036-1044.

424 Jin Y, Mazza C, Christie JR, Giliani S, Fiorini M, Mella P, Gandellini F, Stewart DM, Zhu Q,

425 Nelson DL, Notarangelo LD, and Ochs HD. 2004. Mutations of the Wiskott-Aldrich Syndrome

426 Protein (WASP): hotspots, effect on transcription, and translation and phenotype/genotype

427 correlation. Blood 104:4010-4019. 10.1182/blood-2003-05-1592

428 Kim AS, Kakalis LT, Abdul-Manan N, Liu GA, and Rosen MK. 2000. Autoinhibition and 429 activation mechanisms of the Wiskott-Aldrich syndrome protein. Nature 404:151-158.

$430 \quad 10.1038 / 35004513$

431 Konstantinidis DG, Pushkaran S, Johnson JF, Cancelas JA, Manganaris S, Harris CE, Williams 432 DA, Zheng Y, and Kalfa TA. 2012. Signaling and cytoskeletal requirements in erythroblast 433 enucleation. Blood 119:6118-6127. 10.1182/blood-2011-09-379263 
434 Looi CY, Sasahara Y, Watanabe Y, Satoh M, Hakozaki I, Uchiyama M, Wong WF, Du W, 435 Uchiyama T, Kumaki S, Tsuchiya S, and Kure S. 2014. The open conformation of WASP 436 regulates its nuclear localization and gene transcription in myeloid cells. Int Immunol 26:341437 352. 10.1093/intimm/dxt072

438 Machesky LM, and Insall RH. 1998. Scar1 and the related Wiskott-Aldrich syndrome protein, 439 WASP, regulate the actin cytoskeleton through the Arp2/3 complex. Curr Biol 8:1347-1356. 440 Massaad MJ, Ramesh N, and Geha RS. 2013. Wiskott-Aldrich syndrome: a comprehensive 441 review. Ann N Y Acad Sci 1285:26-43. 10.1111/nyas.12049

442 Matalon O, Reicher B, and Barda-Saad M. 2013. Wiskott-Aldrich syndrome protein--dynamic 443 regulation of actin homeostasis: from activation through function and signal termination in $\mathrm{T}$ 444 lymphocytes. Immunol Rev 256:10-29. 10.1111/imr.12112

445 Misu S, Takebayashi M, and Miyamoto K. 2017. Nuclear Actin in Development and 446 Transcriptional Reprogramming. Front Genet 8:27. 10.3389/fgene.2017.00027

447 Nurnberg A, Kitzing T, and Grosse R. 2011. Nucleating actin for invasion. Nat Rev Cancer 448 11:177-187. 10.1038/nrc3003

449 Parolini O, Berardelli S, Riedl E, Bello-Fernandez C, Strobl H, Majdic O, and Knapp W. 1997. 450 Expression of Wiskott-Aldrich syndrome protein (WASP) gene during hematopoietic 451 differentiation. Blood 90:70-75.

452 Patel VP, and Lodish HF. 1987. A fibronectin matrix is required for differentiation of murine 453 erythroleukemia cells into reticulocytes. J Cell Biol 105:3105-3118.

454 Rotty JD, and Bear JE. 2014. Competition and collaboration between different actin assembly 455 pathways allows for homeostatic control of the actin cytoskeleton. Bioarchitecture 5:27-34. $456 \quad 10.1080 / 19490992.2015 .1090670$ 
457 Rotty JD, Wu C, Haynes EM, Suarez C, Winkelman JD, Johnson HE, Haugh JM, Kovar DR, and 458 Bear JE. 2015. Profilin-1 serves as a gatekeeper for actin assembly by Arp2/3-dependent and 459 independent pathways. Dev Cell 32:54-67. 10.1016/j.devcel.2014.10.026

460 Sadhukhan S, Sarkar K, Taylor M, Candotti F, and Vyas YM. 2014. Nuclear role of WASp in 461 gene transcription is uncoupled from its ARP2/3-dependent cytoplasmic role in actin

462 polymerization. J Immunol 193:150-160. 10.4049/jimmunol.1302923

463 Sakuma C, Sato M, Takenouchi T, Chiba J, and Kitani H. 2012. Critical roles of the WASP N464 terminal domain and Btk in LPS-induced inflammatory response in macrophages. PLoS One 465 7:e30351. 10.1371/journal.pone.0030351

466 Sakuma C, Sato M, Takenouchi T, and Kitani H. 2015. Specific binding of the WASP N467 terminal domain to Btk is critical for TLR2 signaling in macrophages. Mol Immunol 63:328-336.

468 10.1016/j.molimm.2014.08.004

469 Suarez C, Carroll RT, Burke TA, Christensen JR, Bestul AJ, Sees JA, James ML, Sirotkin V, and 470 Kovar DR. 2015. Profilin regulates F-actin network homeostasis by favoring formin over Arp2/3 471 complex. Dev Cell 32:43-53. 10.1016/j.devcel.2014.10.027

472 Symons M, Derry JM, Karlak B, Jiang S, Lemahieu V, McCormick F, Francke U, and Abo A. 473 1996. Wiskott-Aldrich syndrome protein, a novel effector for the GTPase CDC42Hs, is 474 implicated in actin polymerization. Cell 84:723-734.

475 Thrasher AJ, and Burns SO. 2010. WASP: a key immunological multitasker. Nat Rev Immunol $476 \quad 10: 182-192.10 .1038 /$ nri2724

477 Zhu Q, Watanabe C, Liu T, Hollenbaugh D, Blaese RM, Kanner SB, Aruffo A, and Ochs HD. 478 1997. Wiskott-Aldrich syndrome/X-linked thrombocytopenia: WASP gene mutations, protein 479 expression, and phenotype. Blood 90:2680-2689. 
481

482 
483

484

485

486

487

488

489

490

491

492

493

494

495

496

497

498

499

500

501

502

503

504

\section{LEGENDS OF FIGURES}

Figure 1. WASp is poorly expressed in MEL-R cells. A) Immunoblot analysis of whole-cell extracts from erythroleukemia-resistant cells (MEL-R), MEL cells undifferentiated $(0 \mathrm{~h})$ and differentiated with hexamethylene bisacetamide (HMBA) (120 h), and 3T3 fibroblasts. Equal amounts of protein $(30 \mu \mathrm{g})$ were fractionated by SDS-polyacrylamide gel electrophoresis and analyzed by immunoblotting with an anti-Was antibody. $\alpha$-tubulin was used as a loading control.

B) Immunoblot analysis of whole-cell lysates from stable transfectants overexpressing Was

(MEL-R/ Was $(+)$ processed as in A). Numbers above the panel correspond to clones $8,9,10,11$, 13 and 15. MEL cells and MEL-R cells transfected with an empty vector (C) were treated and analyzed under similar conditions. $\alpha$-tubulin was used as a loading control.

\section{Figure 2. Overexpression of Was induces organization and polymerization of actin} cytoskeleton in MEL-R cells. Immunofluorescence staining of MEL cells, MEL-R/Was (+) transfectants 9, 10 and 11, and MEL-R cells with a mouse monoclonal anti- $\beta$-actin antibody (red). Nuclei were visualized with DAPI (blue). Magnified views indicated by white boxed areas are shown below second-row panels. The scale bar represents $10 \mu \mathrm{m}$.

\section{Figure 3. Overexpression of Was enhances the formation of F-actin in MEL-R}

transfectants. A) Total actin expression was evaluated in MEL-R/Was (+) clones 9, 10 and 11, and MEL-R cells by immunoblotting with an antibody against $\beta$-actin. $\alpha$-tubulin was used as a loading control. B) G-actin and F-actin from MEL-R and MEL-R/Was (+) transfectants 9, 10 and 
505 11, separated after ultracentrifugation (G-actin remains in the supernatant, F-actin found in the 506 pellet) were immunoblotted and probed as in A).

507

508 Figure 4. Deletion of Was in MEL cells using CRISPR/Cas9. A) Genomic map of Was in 509 mouse chromosome X:7658591-7667617, including exons (blue rectangles) and 5' and 3'

510 untranslated regions (red rectangles). sgRNA positions in the genome are shown as vertical

511 discontinued red lines. The sgRNA sequences are highlighted in purple and illustrate the Cas9

512 cleavage region. B) PCR analysis for screening biallelic deletion clones using primers listed in

513 Table S2. PCR products (for clone 1) of the non-deletion amplicon (ND) and the deletion

514 amplicon (D) were electrophoresed on a 1\% agarose gel and stained with ethidium bromide. C)

515 Immunoblotting of total lysates from MEL, MEL/Was ${ }^{-/-}$clones 1,4 and 73, and MEL-R cells. $\alpha-$

516 tubulin was used as a protein loading control.

517

518 Figure 5. Deletion of Was provokes defects in the organization and polymerization of actin.

519 Confocal images showing actin stained with a mouse monoclonal anti- $\beta$-actin antibody (red).

520 Nuclei were visualized with DAPI (blue). Forced expression of $W a s$ in MEL/Was ${ }^{-/-}$clone1 was

521 performed by transient transfection with pcDNA3.1-Was (column 3). Magnified views indicated

522 by white boxed areas are shown below second-row panels. Scale bar represents $10 \mu \mathrm{m}$.

523

524 Figure 6. G-/F-actin ratio is altered in $\mathbf{M E L} /$ Was $^{-/-}$cells. A) Whole-cell lysates from MEL,

$525 \mathrm{MEL} / \mathrm{Was}^{-/}$and MEL-R cells were analyzed by immunoblotting with an antibody against $\beta$ -

526 actin. $\alpha$-tubulin was used as a loading control. B) G-actin and F-actin from MEL and MEL-/Was

$527^{-/}$, separated after ultracentrifugation, were immunoblotted and probed as in A). 
528

\section{Figure 7. Induction of Was expression in MEL-R cells stimulates Btk expression.}

530 Immunoblotting of whole-cell lysates from MEL, MEL/Was ${ }^{-/}$(clones 1 and 73), MEL-R/Was

$531(+)$ and MEL-R cells with a mouse monoclonal anti-Btk-antibody. Ectopic expression of Was by 532 transient transfection with the pcDNA3.1-Was vector marked (+) for clones MEL Was-/- 1 and 53373 and MEL-R $\operatorname{Was}(+)$ 9, 10 and 11. $\alpha$-tubulin was used as a protein loading control.

534

535 Figure 8. Induction of Was expression in MEL-R cells stimulates Btk expression. Confocal 536 immunofluorescence images of MEL, MEL/Was ${ }^{-/}$(clone1), MEL-R/Was (+) (clones 9, 10 and 537 11) and MEL-R cells stained with an anti-Btk monoclonal antibody (green). Nuclear DNA was 538 stained with DAPI (blue). Selected cells of each samples in white boxes areas are amplified 539 below. Scale bar represents $10 \mu \mathrm{m}$. 


\section{Figure 1}

WASp is poorly expressed in MEL-R cells

A) Immunoblot analysis of whole-cell extracts from erythroleukemia-resistant cells (MEL-R), MEL cells undifferentiated $(0 \mathrm{~h})$ and differentiated with hexamethylene bisacetamide (HMBA) $(120 \mathrm{~h})$, and 3T3 fibroblasts. Equal amounts of protein $(30 \mu \mathrm{g})$ were fractionated by SDSpolyacrylamide gel electrophoresis and analyzed by immunoblotting with an anti-Was antibody. a-tubulin was used as a loading control. B) Immunoblot analysis of whole-cell lysates from stable transfectants overexpressing Was (MEL-R/Was(+) processed as in A). Numbers above the panel correspond to clones 8, 9, 10, 11, 13 and 15. MEL cells and MEL-R cells transfected with an empty vector (C) were treated and analyzed under similar conditions. a-tubulin was used as a loading control. 


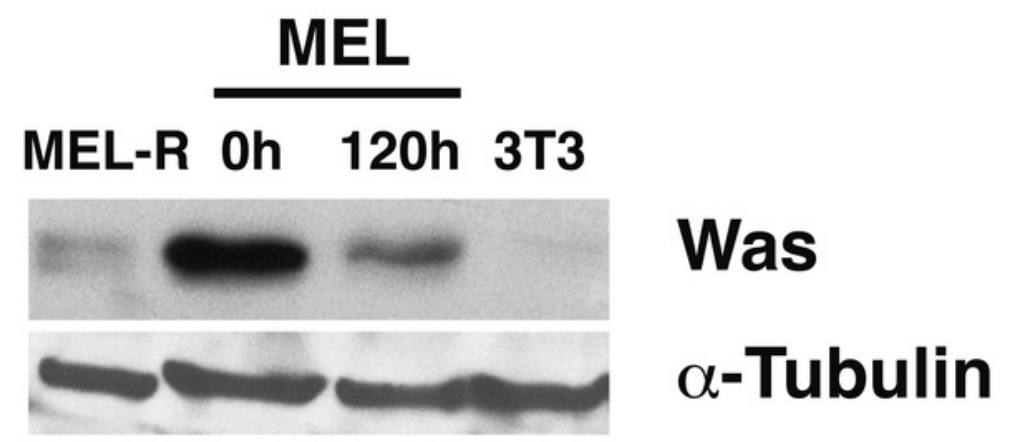

B

\section{MEL-R/ Was (+)}

MEL C

$\begin{array}{lllll}9 & 10 & 11 & 13 & 15\end{array}$

-

Was

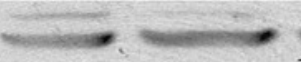

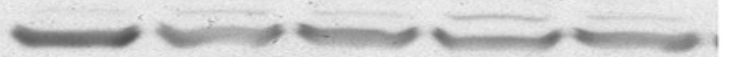

$\alpha$-Tubulin 


\section{Figure 2}

Overexpression of Was induces organization and polymerization of actin cytoskeleton in MEL-R cells

Immunofluorescence staining of MEL cells, MEL-R/Was(+) transfectants 9, 10 and 11, and MEL-R cells with a mouse monoclonal anti-b-actin antibody (red). Nuclei were visualized with DAPI (blue). Magnified views indicated by white boxed areas are shown below second-row panels. The scale bar represents $10 \mathrm{~mm}$.

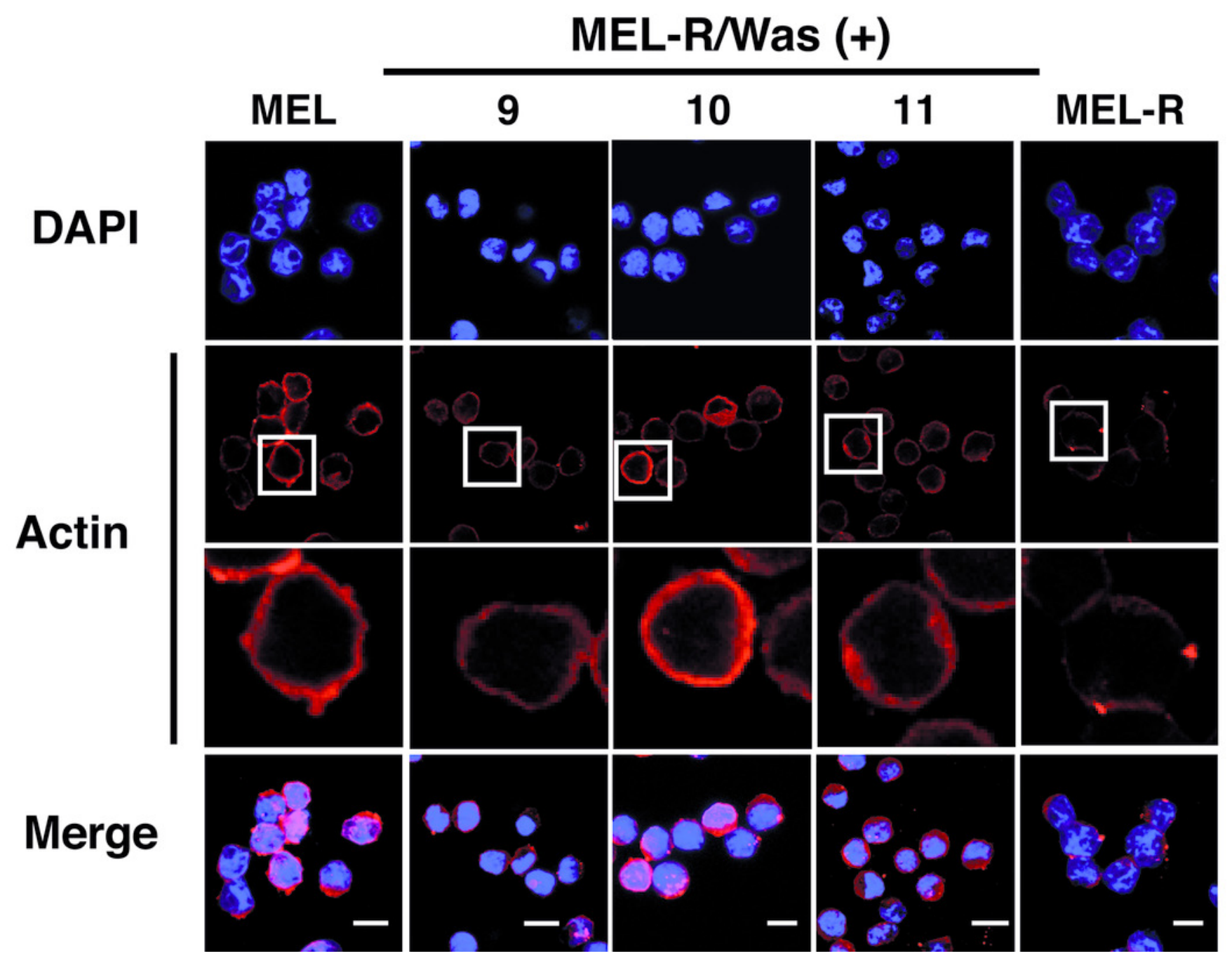




\section{Figure 3}

Overexpression of Was enhances the formation of F-actin in MEL-R transfectants

A) Total actin expression was evaluated in MEL-R/Was(+) clones 9, 10 and 11, and MEL-R cells by immunoblotting with an antibody against b-actin. $\alpha$-tubulin was used as a loading control. B) G-actin and F-actin from MEL-R and MEL-R/Was(+) transfectants 9, 10 and 11, separated after ultracentrifugation (G-actin remains in the supernatant, F-actin found in the pellet) were immunoblotted and probed as in A).

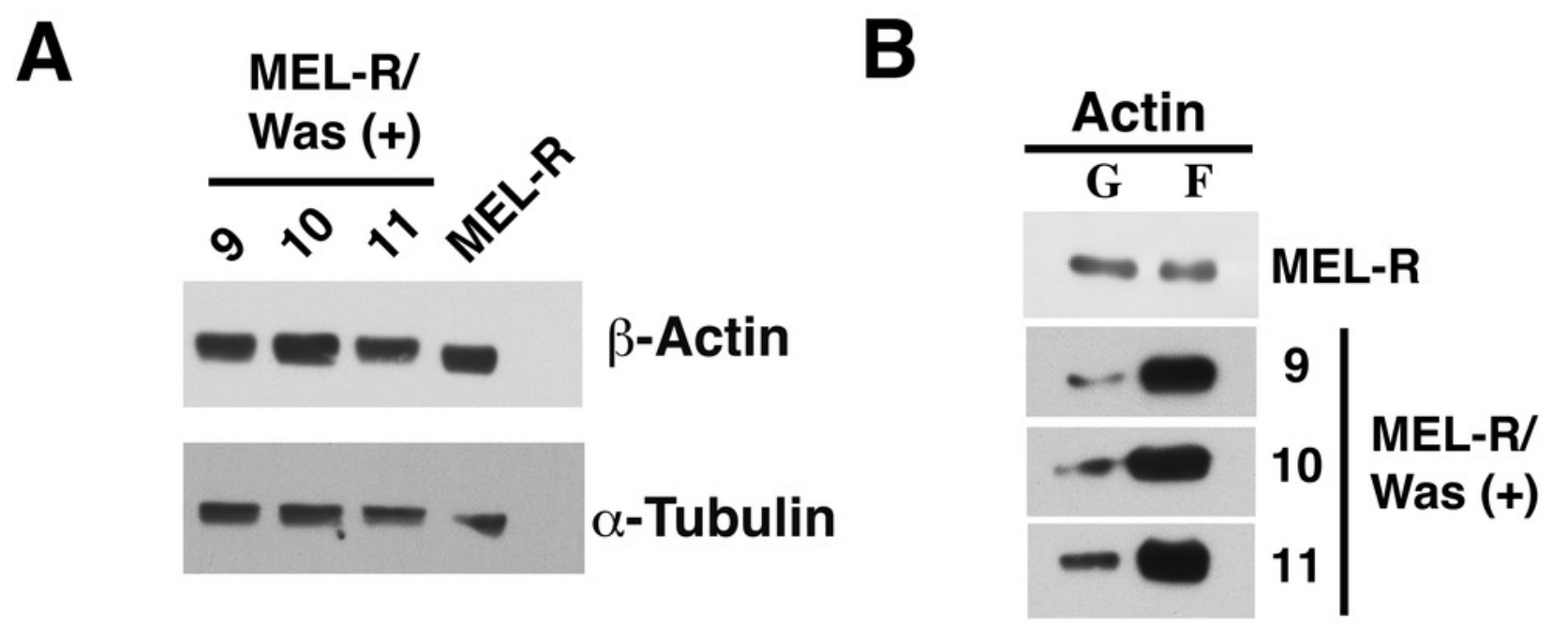




\section{Figure 4}

Deletion of Was in MEL cells using CRISPR/Cas9

A )Genomic map of Was in mouse chromosome X:7658591-7667617, including exons (blue rectangles) and $5^{\prime}$ and $3^{\prime}$ untranslated regions (red rectangles). sgRNA positions in the genome are shown as vertical discontinued red lines. The sgRNA sequences are highlighted in purple and illustrate the Cas9 cleavage region. B) PCR analysis for screening biallelic deletion clones using primers listed in Fig. S3. PCR products (for clone 1) of the non-deletion amplicon (ND) and the deletion amplicon (D) were electrophoresed on a 1\% agarose gel and stained with ethidium bromide. C) Immunoblotting of total lysates from MEL, MEL/Was ${ }^{-/}$clones $^{-}$ 1, 4 and 73, and MEL-R cells. a-tubulin was used as a protein loading control. 
A

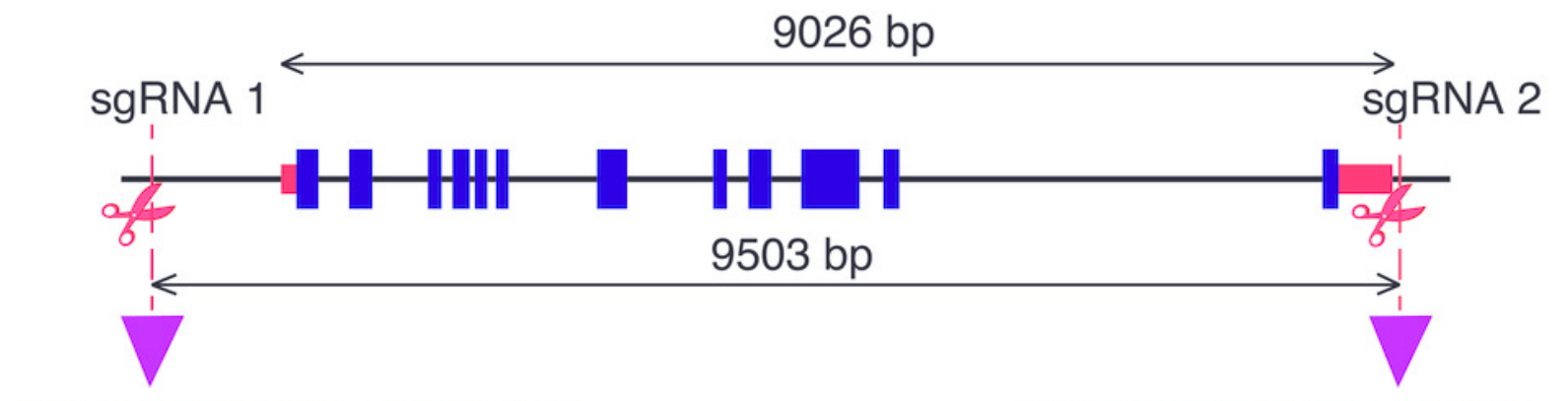

GCCACCACGCCCGGCTCAATATGCCTTTAAA- - - - - - - - - - - - - GCCACGTCGCCTGTATTTCTTTACTTTTCC CGGTGGTGCGGGCCGAGTTATACGGAAATTT- - - - - - - - - - - - - - CGGTGCAGCGGACATAAAGAAATGAAAAGG

B

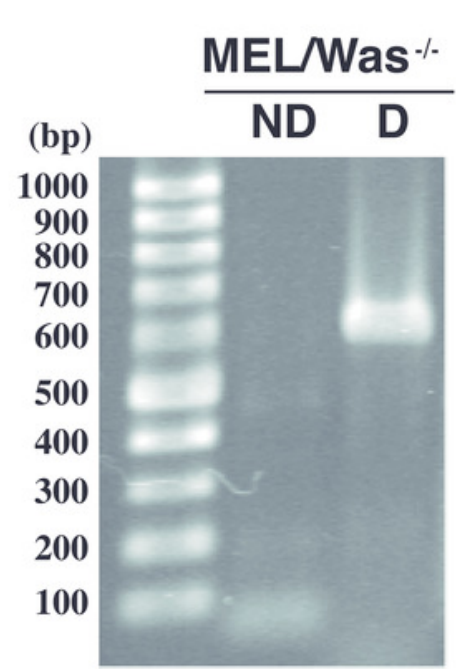

C

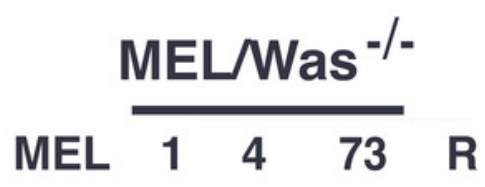

Was

$\alpha-$ Tubulin 


\section{Figure 5}

Deletion of Was provokes defects in the organization and polymerization of actin Confocal images showing actin stained with a mouse monoclonal anti-b-actin antibody (red). Nuclei were visualized with DAPI (blue). Forced expression of Was in MEL/Was ${ }^{-1}$ clone1 was performed by transient transfection with pcDNA3.1-Was (column 3). Magnified views indicated by white boxed areas are shown below second-row panels. Scale bar represents 10 $\mathrm{mm}$.

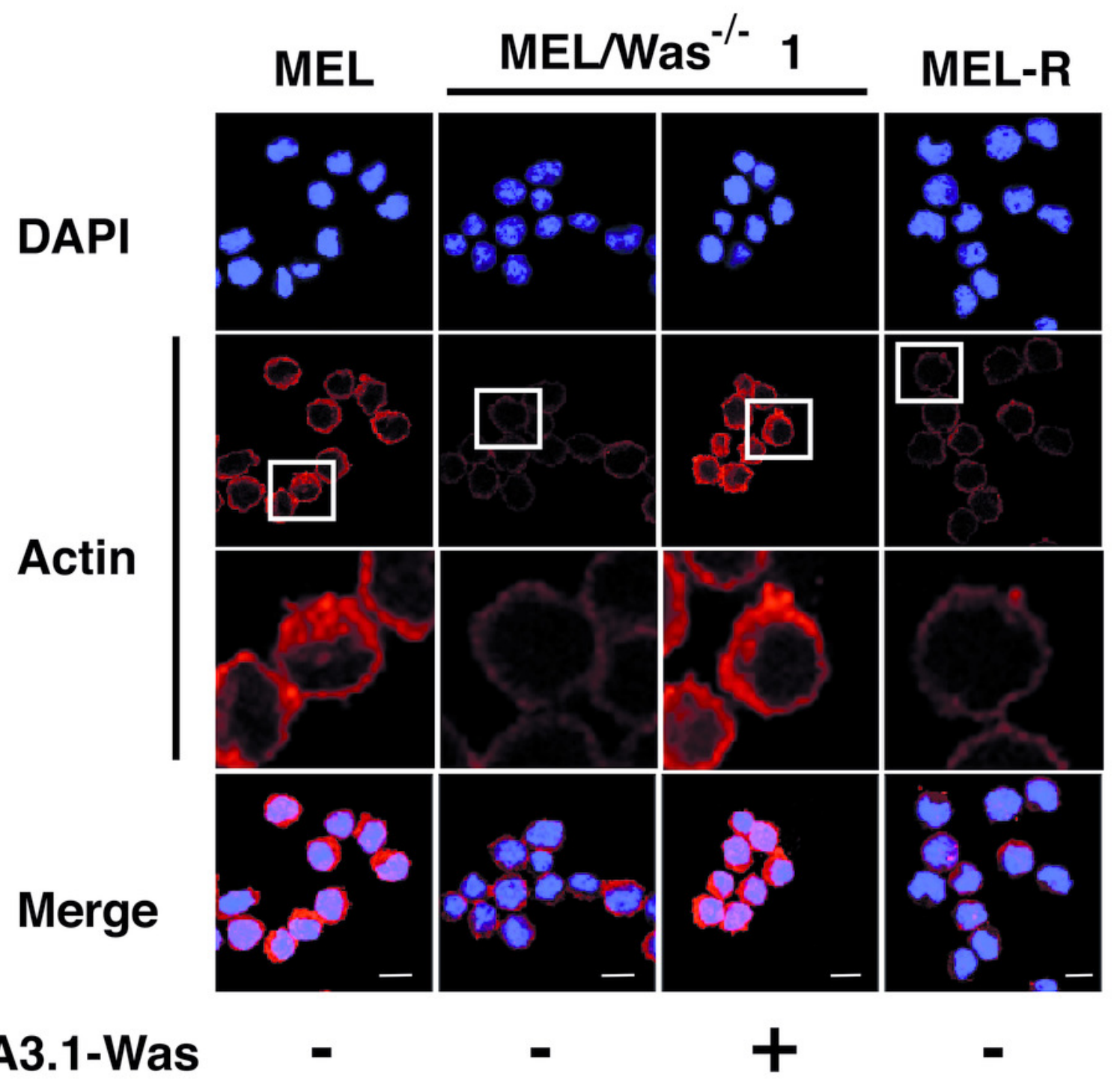




\section{Figure 6}

G-/F-actin ratio is altered in MEL/Was ${ }^{-1}$ cells

A) Whole-cell lysates from MEL, MEL/Was ${ }^{-1}$ and MEL-R cells were analyzed by immunoblotting with an antibody against b-actin. $\alpha$-tubulin was used as a loading control. B) G-actin and Factin from MEL and MEL-/Was ${ }^{-1}$, separated after ultracentrifugation, were immunoblotted and probed as in A).

A

B

MEL
MEL Was'- MEL-R

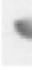$$
=\beta \text {-Actin }
$$

$\alpha$-Tubulin

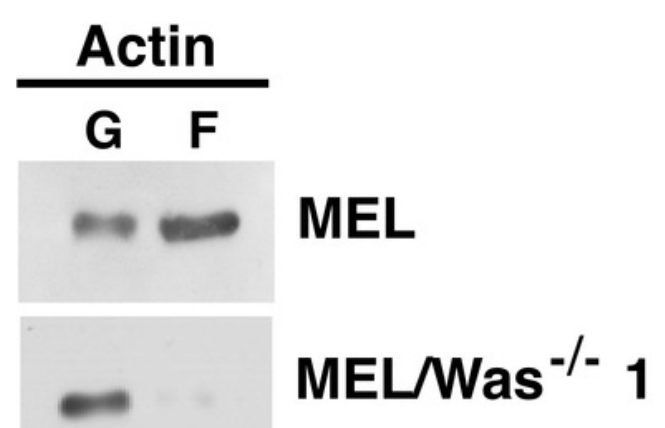




\section{Figure 7}

Induction of Was expression in MEL-R cells stimulates Btk expression

Immunoblotting of whole-cell lysates from MEL, MEL/Was ${ }^{-1}$ (clones 1 and 73), MEL-R/Was (+) and MEL-R cells with a mouse monoclonal anti-Btk-antibody. Ectopic expression of Was by transient transfection with the pcDNA3.1-Was vector marked (+) for clones MEL Was-/- 1 and 73 and MEL-R Was(+) 9, 10 and 11. a-tubulin was used as a protein loading control

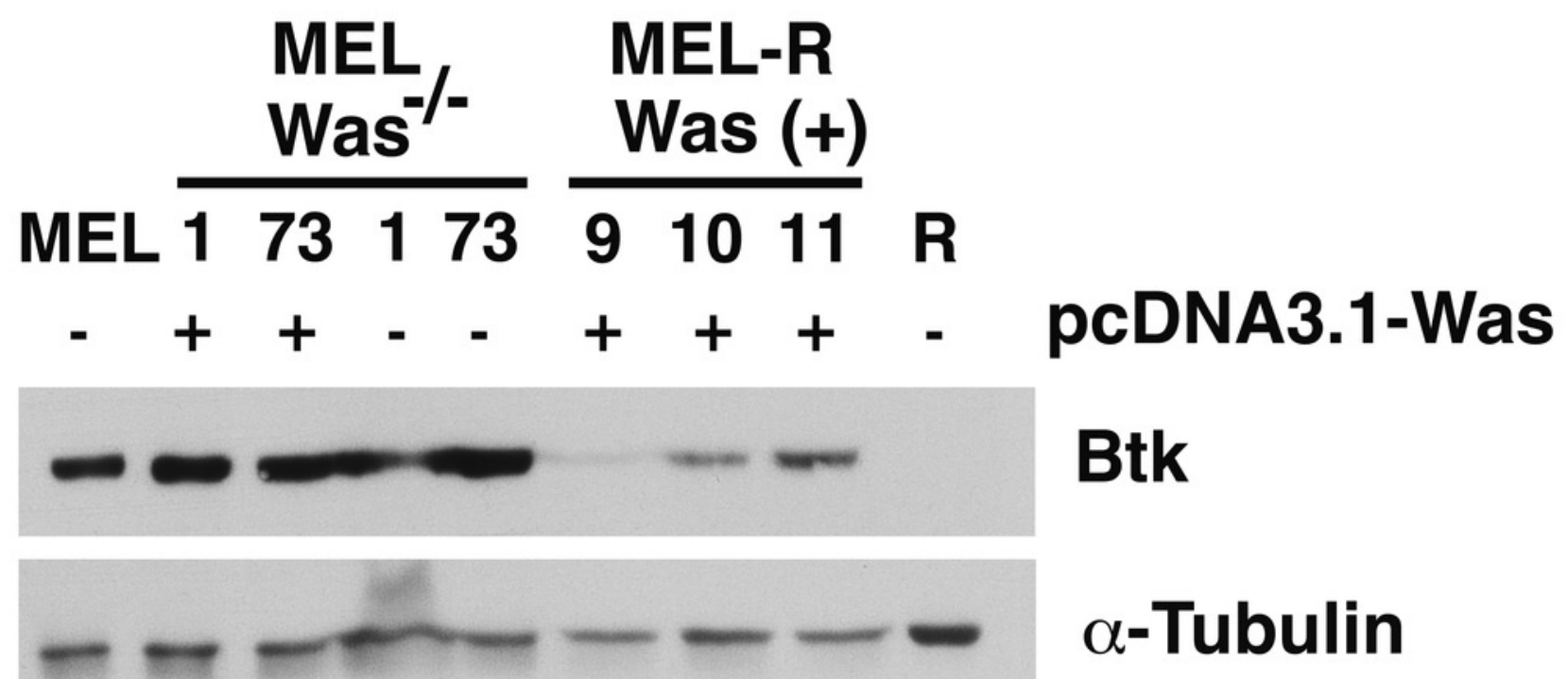




\section{Figure 8}

Induction of Was expression in MEL-R cells stimulates Btk expression

Confocal immunofluorescence images of MEL, MEL/Was ${ }^{-1}$ (clone1), MEL-R/Was (+) (clones 9, 10 and 11) and MEL-R cells stained with an anti-Btk monoclonal antibody (green). Nuclear DNA was stained with DAPI (blue). Selected cells of each samples in white boxes areas are amplified below. Scale bar represents $10 \mathrm{~mm}$.

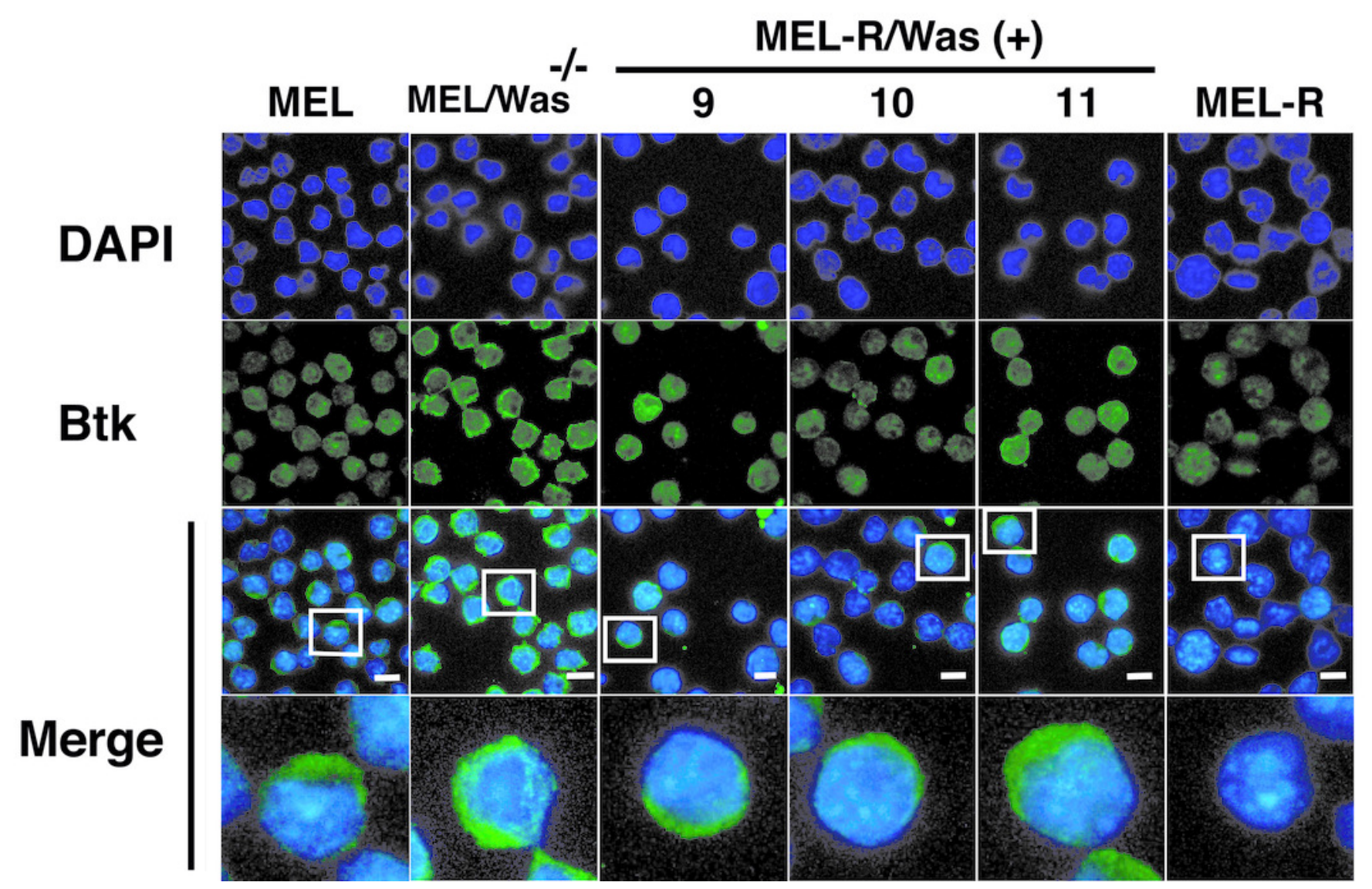

